\title{
The Atlas Tile Calorimeter Road to Physics
}

\section{Agostinho Gomes*}

on behalf of the Tile Calorimeter subsystem of the ATLAS Collaboration

LIP and CFNUL, Lisbon, Portugal

E-mail: agomes@lip.pt

The Tile Calorimeter is the barrel hadronic calorimeter of the ATLAS detector, covering the region $|\eta|<1.7$. It is a non-compensating sampling device made of plastic scintillator embedded in an iron structure. The construction phase of the calorimeter is almost complete, and most of the effort now is directed towards the final assembly and commissioning in the underground experimental hall. The central part of the barrel is already assembled and the electronics is in installation and commissioning phase.

The design requirements and layout of the calorimeter are presented, as well as its expected performance for jets. Detector calibration and commissioning are briefly discussed, showing the first cosmic events taken by the Tile Calorimeter in the ATLAS cavern during commissioning.

During the last few years a comprehensive testbeam program has been followed in order to establish the calorimeter electromagnetic energy scale, to study its uniformity, and to tune the Monte Carlo simulations. During the combined testbeam period in 2004, lasting several months, data have been acquired with a complete slice of the central ATLAS barrel, inner and muon detectors, as well as both calorimeters. The data collected in the testbeam are used to study algorithms for hadronic energy reconstruction using single particles and the generalization of these algorithms is crucial for the jet reconstruction. Preliminary results on detector performance such as energy resolution and linearity are presented.

International Europhysics Conference on High Energy Physics

July 21st - 27th 2005

Lisbon, Portugal

\footnotetext{
${ }^{*}$ Speaker.
} 


\section{Introduction and construction}

The Tile Calorimeter of ATLAS is a large sampling calorimeter which makes use of steel as absorber and scintillating tiles read out by wavelength shifting fibres (WLS) as active material. The new feature of its design is the orientation of the scintillating tiles which are placed in planes perpendicular to the colliding beams and staggered in depth [1]. The WLS fibres running radially collect the light from the tiles along the two open edges. Readout cells are defined by grouping together a set of fibres into a photomultiplier (PMT). The calorimeter is segmented in three layers with thickness $1.5,4.1$ and 1.8 interaction lengths at $\eta=0$. The $\Delta \eta \times \Delta \phi$ is $0.1 \times 0.1$ (and $0.2 \times$ 0.1 in the outer radial layer). The Tile Calorimeter is composed of 3 cylinders, one $\sim 6 \mathrm{~m}$ long Central Barrel covering the $\eta$ region up to 1.0 and two Extended Barrels extending the $\eta$ coverage up to 1.7. Each cylinder have a $2.28 \mathrm{~m}$ inner radius and a $4.25 \mathrm{~m}$ outer radius and is composed of 64 modules. The PMTs and part of the front end electronics are located in drawers that go inside the girder located in the outer side of the modules. The Tile Calorimeter is located behind the Liquid Argon (LArg) electromagnetic (em) calorimeter. Globally, the design requirements to the ATLAS hadron calorimeters have been good hermeticity and $\eta$ coverage up to 5 for a good missing transverse energy measurement, and a jet energy resolution of the order of $50 \% / \sqrt{E} \oplus 3 \%$ with a non-linearity better than a few percent up to a few TeV.

The production of the Tile Calorimeter modules started in 1998. After the production of the modules, one Extended Barrel and the Central Barrel cylinders were assembled on the surface at CERN in order to prepare the work for the final assembly in the cavern. This pre-assembly allowed the test and certification of tools and supports, deformations measurements, confirmation of shims dimensions and load tests of the LArg calorimeter weight.
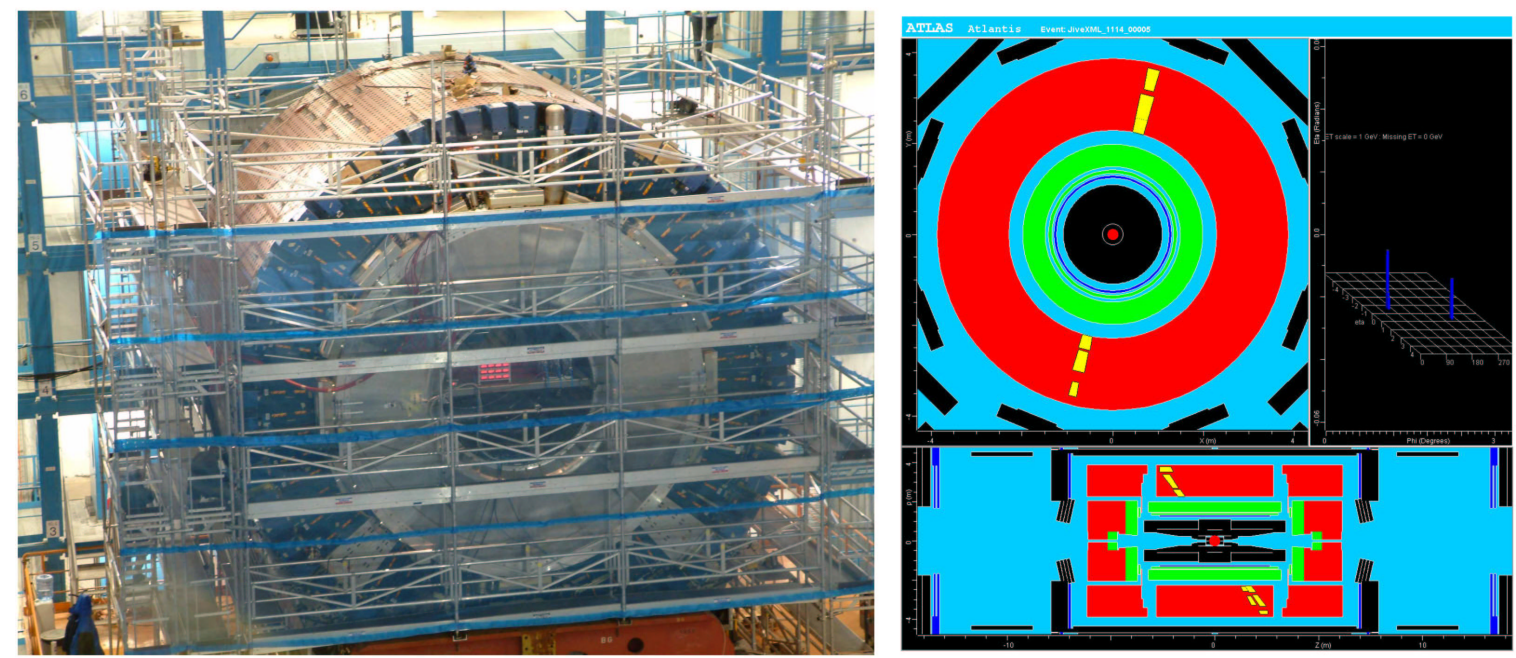

Figure 1: Left - The Central Barrel Tile and LArg calorimeters assembled in the ATLAS cavern. Right One of the first cosmic muon events recorded by the Tile Calorimeter in the ATLAS cavern.

The first modules were lowered to the cavern in February 2004. The assembly of the Central Barrel cylinder with the LArg em calorimeter inside was finished in December 2004 (figure 1-left). After the end of the mechanical assembly, the services installation started as well as the certification of the electronics inside the drawers and the installation of power supplies, DAQ and DCS. 

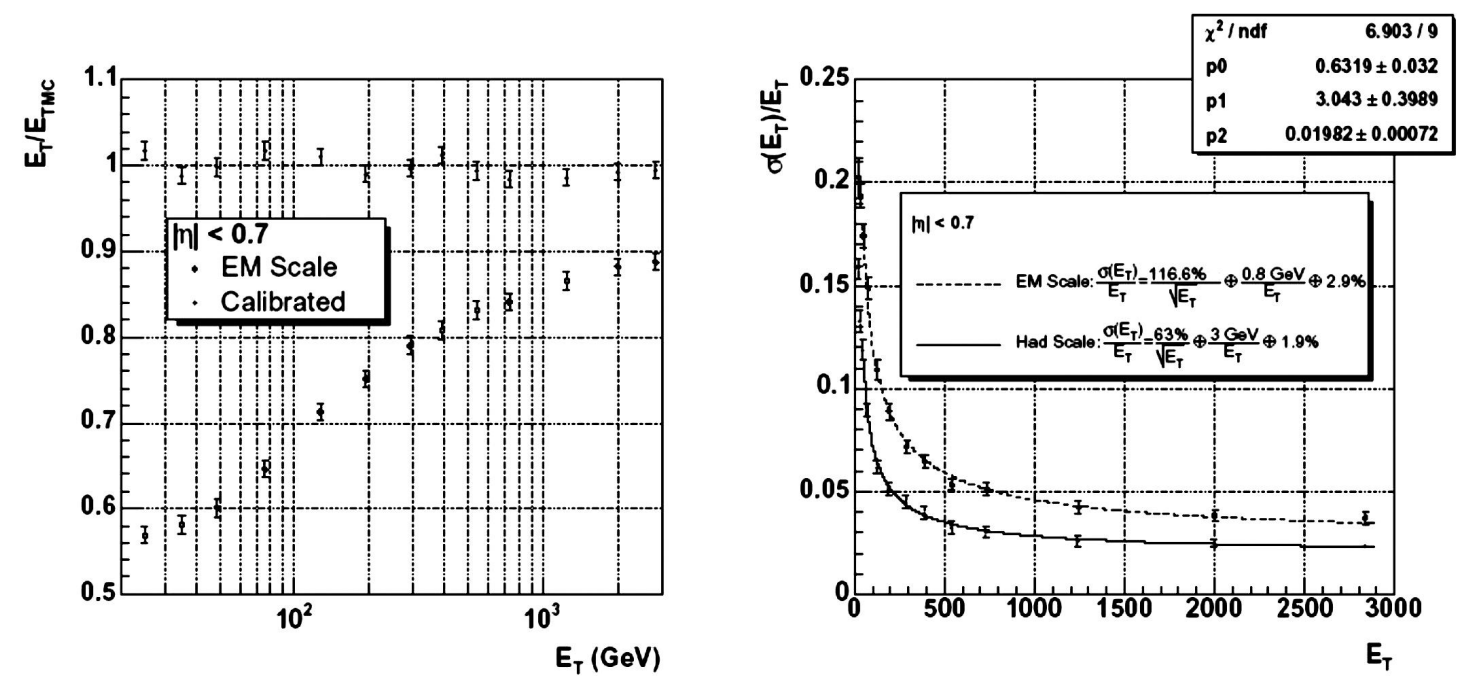

Figure 2: Linearity (left) and resolution (right) for QCD dijet events transverse energy at electromagnetic scale and after calibration. The simulated events include electronic noise.

\section{Testbeam and commissioning}

A fraction of the production modules equipped with electronics, was exposed to high energy particles in the SPS beam line H8 at CERN in order to establish the calorimeter em energy scale, to study its uniformity, and to tune the Monte Carlo (MC) simulations. During 2004 a combined testbeam took place including all the sub-detectors of ATLAS making a vertical slice of the detector. In this period the central em and hadronic calorimeters were tested together in the ATLAS configuration. The analysis of the data taken is underway and it will take some time until final combined results are available. Preliminary analysis of pions showering only in the Tile Calorimeter (requiring mip in the em LArg calorimeter in front) shows energy resolutions compatible with the ones obtained in 1997 and 1998 standalone testbeams, e.g., in the Barrel at $\eta=0.45$ the raw energy resolution parametrization obtained in 2004 is $\sigma / E=54 \% / \sqrt{E} \oplus 5.4 \%$ compared with $\sigma / E=57 \% / \sqrt{E} \oplus 5.4 \%$ in the standalone tests [2]. After the assembly of the Central Barrel cylinder in the ATLAS cavern, the commissioning of the detector started. Special trigger boards were prepared and it was possible to detect the first cosmic muons in June 2005. An example of a cosmic muon event that crosses the Tile Calorimeter is displayed in figure 1-right.

\section{Hadronic energy reconstruction}

The non-compensation of the ATLAS calorimeters implies that the energy measured for jets needs to be corrected. One way to do the correction is to compute weights for the energies of the cells associated to a jet. One of the methods used has been developed by the H1 experiment [3] and implementations of these cell weighting methods have been tested in ATLAS [4]. The jet energy is defined as the sum of the raw energies of the cells belonging to the jet multiplied by a weight that depends on the cell energy and on the jet energy: $E_{J}^{r e c}=\sum w_{i}\left(E_{i}, E_{J}\right) E_{i}$. The weights $w_{i}$ for each cell are function of the energy deposited in the cell $E_{i}$ and of the jet energy $E_{J}$, being used for the jet energy the true MC energy $E_{M C}$ in the simulation case. The weights are obtained by minimizing the energy resolution with the constraint that the total reconstructed energy after re-weighting is equal 
to the true MC energy. This method was already used on testbeam and simulated data and is able to restore the linearity and to improve the energy resolution [4][5]. The results obtained for samples of simulated QCD dijet events in the central region of ATLAS $(|\eta|<0.7)$ are shown in figure 2, where it can be noticed the significative improvements in the jets $E_{T}$ linearity and resolution when the calibration is applied.

Since in the real data the value of the true energy is not available, the jet energy has to be calculated with an iterative procedure and using only measurable quantities. The unweighted sum of the cell energies is used for the initial estimate of the weights, then the calibrated energy obtained is used as input to calculate new weights and new estimate of the energy and after a few iterations the reconstructed energy changes from one iteration to the next one become small. The application of the iterative procedure to the jets gives practically the same linearity as shown in figure 2 , and the $E_{T}$ resolution is slightly worse than in the case where we knew the true jet energy: $\sigma / E_{T}=$ $61 \% / \sqrt{E_{T}} \oplus 4.8 \mathrm{GeV} / E_{T} \oplus 2.0 \%$ [5]. The study of these promising weighting techniques is in progress.

\section{Conclusions}

By the end of 2004, the central barrel of the Tile Calorimeter was assembled in the ATLAS cavern at CERN. The installation of the front end electronics is in progress and the low voltage power supplies are in production. A limited number of modules in the cavern was already fully equipped with electronics and cosmic ray muons were detected using a trigger board developed specifically for this purpose. The data obtained in the combined testbeam during 2004 is being analysed in order to understand the behaviour of the full detector and in particular the combined calorimeter. At the same time, offline software development continues, including algorithms for jet energy reconstruction, that will be tested with MC data and also with the data acquired in the combined testbeam. The Tile Calorimeter construction phase is nearly finished, the assembly and commissioning in the cavern is proceeding, and preparations are underway to analyze first collision data when available in 2007.

\section{Acknowledgements}

I would like to thank T. Davidek, A. Henriques, J. Maneira, C. Roda, J. Saraiva, R. Stanek and I. Vivarelli for the help in the preparation of the talk and all the ATLAS colleagues that contributed to the construction of the detector, to the testbeams and to the physics simulation. This work was supported in part by Fundação para a Ciência e Tecnologia within the project POCI/FP/63422/2005.

\section{References}

[1] F. Ariztizabal et al, Nucl. Instrum. Methods A349 (1994) 384; ATLAS Tile Calorimeter TDR, CERN/LHCC/96-42, 1996

[2] V. Giangiobbe, presentation in ATLAS Physics workshop, Rome, 8 Jun 2005

[3] I. Abt et al, Nucl. Instrum. Methods A386 (1997) 348

[4] M. P. Casado and M. Cavalli-Sforza, ATLAS note ATL-TILECAL-96-075, 1996;

S. Akhmadaliev et al, Nucl. Instrum. Methods A449 (2000) 461

[5] C. Roda and I. Vivarelli, Cell Based Jet Calibration, ATLAS note ATL-PHYS-PUB-2005-019, 2005 